\section{Atopisches Ekzem: Cave COX-2-Inhibitoren!}

\author{
Spezifische Hemmer der Cyclooxygenase 2 verursachen weniger \\ gastrointestinale Nebenwirkungen als klassische nicht steroidale \\ Antiphlogistika. Wissenschaftler aus Boston untersuchten jetzt den \\ Einfluss der Cyclooxygenase 2 auf die allergische Hautreaktion.
}

\begin{abstract}
E orscher der Harvard Medical School verletzten die Haut von Mäusen oberflächlich und induzierten mit Hilfe eines sensibilisierenden OvalbuminPflasters eine epikutane Entzündung, die dem menschlichen atopischen Ekzem ähnelt: Sie ist charakterisiert durch eine eosinophile Hautinfiltration, eine Erhöhung von spezifischem IgE und GesamtIgE und eine systemische TH2-Antwort nach Antigenprovokation. Erhielten die Mäuse zusätzlich den Cyclooxygenase(COX-)2-Hemmer NS-398, waren die eosinophile Hautinfiltration sowie die IL-4-, IgE- und $\mathrm{IgG}_{1}$-Serumspiegel nach
\end{abstract}

Ovalbumin-Provokation signifikant erhöht im Vergleich zu Kontrollmäusen. Auch zeigten sich bei den NS-398-behandelten Nagern eine herabgesetzte Ovalbumin-spezifische $\operatorname{IgG}_{2 \mathrm{a}}$-Antwort sowie eine reduzierte IFN- $\gamma$-Sekretion der Milzzellen. Ein ähnliches Entzündungsmuster in Form einer gesteigerten TH2Antwort ließ sich auch bei COX-2-defizienten Mäusen nach einer epikutanen Ovalbumin-Sensibilisierung nachweisen.

COX-2-Syntheseprodukte haben offensichtlich einen hemmenden Einfluss auf die TH2-Antwort bei einer experimentellen epikutanen Sensibilisie- rung, während durch die Gabe eines COX-2-Inhibitors die TH2-Antwort gesteigert wird. Deshalb könnte die Einnahme von COX-2-Hemmern die entzündliche Hautreaktion bei Patienten mit atopischem Ekzem verstärken. Allerdings können die hier vorgestellten Ergebnisse nicht die Tatsache erklären, dass das COX-2-Produkt Prostaglandin E2 sehr häufig in erhöhten Konzentrationen in der Haut von Neurodermitispatienten nachweisbar ist und in vitro die TH1Zellen hemmt sowie die Etablierung von TH2-Zellen fördert.

Fazit: COX-2-Hemmer können möglicherweise eine allergische Hautentzündung beim atopischen Ekzem verschlimmern

Laouini $D$ et al. COX-2 inhibition enhances the $\mathrm{TH}_{2}$ immune response to epicutaneous sensitization. J Allergy Clin Immunol 2005; 116: 390-6

\section{Neurodermitishaut ist krebsgefährdet}

\author{
Bei Behandlung einer atopischen Dermatitis werden Maßnahmen \\ eingesetzt, die potenziell karzinogen sind. In einer dänischen \\ Kohortenstudie sollte das Krebsrisiko von Patienten mit einer \\ schweren Erkrankung ermittelt werden.
}

A nhand des Danish National Hospital Register wurden alle Fälle einer Krankenhauseinweisung aufgrund der primären Diagnose atopische Dermatitis (AD) zwischen 1977 und 1996 erfasst und anhand des Danish Cancer Register

mit einer möglichen Krebsdiagnose bis zum Jahr 1996 korreliert. Die Daten von 6.275 Personen wurden ausgewertet. Während in der Gruppe der 4.245 Kinder und Jugendlichen keine erhöhte Krebsinzidenz festgestellt wurde, war bei

\begin{tabular}{lccr} 
Tabelle. Krebserkrankungen bei 6.275 Patientren mit atopischem Ekzem \\
\cline { 2 - 3 } & Beobachtet & Erwartet & SMR (95\%-KI) \\
Gesamt & 67 & 44,3 & $1,5(1,2-1,9)$ \\
Gastrointestinaltrakt & 7 & 6,4 & $1,1(0,4-2,2)$ \\
Atmungsorgane & 8 & 4,6 & $1,7(0,7-3,4)$ \\
\hline Mammakarzinom & 10 & 6,9 & $1,4(0,7-2,7)$ \\
Krebs der weiblichen Geschlechtsorgane & 10 & 4,6 & $2,2(1,0-4,0)$ \\
Krebs der männlichen Geschlechtsorgane & 6 & 2,6 & $2,3(0,8-4,0)$ \\
Keratinozyten-Karzinome & 16 & 6,6 & $2,4(1,4-3,9)$ \\
Lymphome und Leukämien & 4 & 2,9 & $1,4(0,4-3,5)$ \\
Andere & 6 & 9,7 & $0,6(0,2-1,3)$ \\
\hline SMR, Standard-Morbiditätsrate; KI, Konfidenzintervall & & &
\end{tabular}

den 2.030 Erwachsenen das tatsächliche Krebsrisiko im Vergleich zum erwarteten Risiko erhöht (Tabelle). Die Hälfte der zusätzlichen Fälle waren von den Keratinozyten ausgehende Karzinome in den ersten neun Jahren des Beobachtungszeitraums.

Die erhöhte Krebsinzidenz könnte in Zusammenhang mit der verwendeten Therapie stehen: So wirken die bei schwerer $\mathrm{AD}$ häufig eingesetzten UVoder Teer-Behandlungen karzinogen. Eine weitere Erklärung könnte aber auch ein Detektionsbias sein: AD-Patienten werden regelmäßig dermatologisch untersucht, was zu einer früheren Hautkrebsdiagnose führen könnte. Auf Patienten mit leichterer AD dürfen die Ergebnisse nicht übertragen werden.

Fazit: Bei erwachsenen Patienten mit einer schweren $\mathrm{AD}$ ist das Risiko für Hautkrebs erhöht. Ein Zusammenhang mit Therapieverfahren wie UV-Bestrahlung oder Teerbädern ist denkbar. $\quad b k$

Olesen AB et al. The risk of cancer among patients previously hospitalized for atopic dermatitis. J Invest Dermatol 2005; 125: 445-9 